ORIGINAL

\title{
Efficacy of combined therapy of goserelin and letrozole on very young women with advanced breast cancer as first-line endocrine therapy
}

\author{
Xiaoyi Liu, Huili Qu, Weihong Cao,Yu Wang, Zhongliang Ma, Funian Li and Haibo Wang \\ Department of Galactophore, the Affiliated Hospital of Medical College, Qingdao University, Qingdao, China
}

\begin{abstract}
Breast cancer in young women younger than 35 years old is rare, aggressive and associated with a poor prognosis. Endocrine therapy is a preferred treatment modality in hormone receptor-positive early stage and advanced breast cancer, combined therapy of goserelin and letrozole presents an option for premenopausal women. We reported the efficacy and safety of therapy of goserelin plus letrozole on very young women with advanced breast cancer as first-line endocrine therapy. Thirty-five patients with first diagnosed as advanced breast cancer, age younger than 35 years, were enrolled in the study. All patients received goserelin $3.6 \mathrm{mg}$ by subcutaneous injection every 4 weeks along with letrozole $2.5 \mathrm{mg}$ daily by mouth as first-line endocrine therapy. The study endpoints were objective response rate (ORR), clinical benefit (CB), progression-free survival (PFS), overall survival (OS) and toxicity. The median duration of response to the therapy was 21 (range, 10-56) months, and median duration of follow-up was 44 (range, 5-79) months. The ORR was $25.7 \%$, with one complete response (CR, 2.9\%) and eight partial response (PR, 22.9\%). Twenty-two patients had stable disease at 24 weeks, for a clinical benefit rate of $65.7 \%$. The median PFS was 9.6 (range 5-58) months and median OS was 33 (range 6-72) months. During the therapy and follow-up, no serious toxicities were reported. Combined therapy of goserelin and letrozole appears to be an efficacious and well-tolerated therapy for very young women with advanced breast cancer. Further investigations involving more patients, combination of other therapies and longer follow-up are requisite.
\end{abstract}

Key words: Advanced breast cancer, Endocrine therapy, Goserelin, Letrozole

\begin{abstract}
ALTHOUGH BREAST CANCER is the most common cancer in women in the developing and developed countries, only $1 \%$ of the patients are younger than 35 years old [1]. Breast cancer in young women is considered aggressive and associated with a poor prognosis $[2,3]$. Earlier data from the Finnish Cancer Registry in the 1990's showed that very young women had a more advanced breast cancer at diagnosis and poorer 5-year survival than older premenopausal patients [4]. Recent data indicated the 5-year overall survival (OS) for young women had been better but is still lower than for all breast cancer patients, and disease free interval (DFI) was shorter in patients with hormone receptor positive disease [1].

Endocrine therapy is the preferred treatment modality in hormone-receptor-positive (HR) early stage and

Submitted Dec. 1, 2012; Accepted Feb. 18, 2013 as EJ12-0434 Released online in J-STAGE as advance publication Mar. 1, 2013

Correspondence to: Haibo Wang, Department of Galactophore, the Affiliated Hospital of Medical College, Qingdao University, NO. 59, Haier Road, Qingdao, China. E-mail: qdyxywhb@163.com

(C) The Japan Endocrine Society
\end{abstract}

advanced breast cancer, and is based on reducing circulating levels of estrogens. Since the ovary is a major contributor of circulatory estrogen, a variety of endocrine therapies revolve around reducing ovarian production or function of estrogen, including selective estrogen receptor modulators (tamoxifen and toremifene), aromatase inhibitors (AIs; anastrozole, letrozole, exemestane), ovarian ablation, ovarian suppression with luteinizing hormone-releasing hormone (LHRH) agonists (goserelin, leuprolide, triptorelin), pure estrogen antagonists (fulvestrant), and progestational agents (megesterol acetate). Nevertheless, for premenopausal women with breast cancer, especially very young women, protection of ovarian function is important in order to maintain quality of life and fertility [5-9].

LHRH analogues decrease ovarian estradiol production indirectly by acting on the hypothalamic-pituitaryovarian axis and inhibiting the secretion of pituitary gonadotrophins. Chronic and reversible suppression of gonadotropin secretion leads to a loss of ovarian steroid production, which is an optimal antitumor environment 
for estrogen-responsive tumors [5, 7]. Goserelin is a widely-used LHRH analogue shown to be effective and well-tolerated in patients with advanced breast cancer. It is also used to decrease gonado-toxicity and prevent premature menopause induced by chemotherapy in young, early breast cancer patients when administered before and during treatment [10] since it has been observed to protect ovarian function from damage by chemotherapy. Goserelin is associated with fewer adverse events and comparative long-term disease-free survival and higher quality of life in patients with ER+ breast cancer compared to chemotherapy alone [11-13].

Aromatase inhibition can restrain peripheral conversion of androgens to estrogens and reduce circulating levels of estrogens by more than $90 \%$ [14]. In patients with advanced breast cancer and known HR status, third-generation aromatase inhibitors (AIs), such as anastrozole, exemestane, and letrozole have proven to be superior to tamoxifen in terms of time to disease progression $[15,16]$. Moreover, letrozole does not affect the levels of other hormones, including cortisol, androstenedione, aldosterone, and thyroid stimulating hormone [17]. For these reasons, letrozole is usually administered as first-line endocrine therapy in advanced disease.

Combination of an LHRH analog and an AI presents an option for premenopausal women. In a study involving premenopausal women with advanced breast cancer, ovarian suppression combined with AIs induced a more intense reduction in estrogen levels than ovarian suppression plus tamoxifen $[6,10]$. According to a phase $I I$ study performed in 16 premenopausal women with advanced breast cancer, the goserelin and anastrozole combination therapy as a second-line endocrine treatment produced a clinical benefit rate of $75 \%$ with a median duration of remission of 17 months [18]. The Austrian Breast and Colorectal Cancer Study Group (ABCSG-12) recently reported results showing that goserelin plus anastrozole yielded clinical outcomes in disease-free survival that were similar to those of goserelin plus tamoxifen in premenopausal women with hormone-responsive early breast cancer, but worse in overall survival [19]. While Masuda reported that more patients in the anastrozole group had a complete or partial response than did those in the tamoxifen group during 24 weeks of neoadjuvant treatment [20].

A recent survey among researchers in the breast cancer field [21] found that the identification of hormone receptor-positive breast cancer patients who might derive equal benefit from endocrine treatment alone without the addition of adjuvant chemotherapy is one of the most important current clinical research topics. Moreover, clinical data from studies carried out in very young women with advanced breast cancer are still limited. Here, we reported a retrospective study that analyzed the efficacy and safety of letrozole plus goserelin in very young patients with advanced breast cancer, as first-line endocrine therapy.

\section{Materials and Methods}

\section{Patients}

Female patients who aged $\leq 35$ years and had been treated with goserelin and letrozole as first-line endocrine therapy for advanced breast cancer from November 2003 to October 2007 in Department of Galactophore, the Affiliated Hospital of Medical College, Qingdao University, were included in the study. All patients were premenopausal and first diagnosed as advanced breast cancer according to their clinical and pathologic manifestation, as defined by WHO classification criteria, with ECOG status 0-2 (Zubrod-ECOG-WHO). Premenopausal status was defined by regular menstruation periods and by ensuring that luteinising hormone $(\mathrm{LH})$, follicle-stimulating hormone (FSH) and oestradiol serum levels were inside the premenopausal range. The patient was considered hormone receptor positive according to the assay results of primary tumor tissue acquired by biopsy. Main exclusion criteria were clinical or radiological signs of central nervous system metastasis; inflammatory breast cancer; other concurrent or previous malignant disease; life-threatening disease, such as uncontrolled cardiac diseases; life expectancy $\leq 6$ months; or a pregnant or lactating status. The study protocol was approved by the Ethical Committee of the Affiliated Hospital of the Medical School, Qingdao University. Written informed consent was obtained from each patient before enrollment in the study.

\section{Tumor biological techniques}

Hormone receptor status, Ki-67 and HER2 expression were determined by immuno- histochemistry on primary tumor material. Four $\mu \mathrm{m}$ sections from whole block tissues were deparaffinised in xylol for 5 min, dehydrated in a graded series of alcohol, and microwave treated for $10 \mathrm{~min}$ in citrate buffer $(\mathrm{pH}$ 6.0). Immunohistochemical evaluation of the ER, PR, 
HER2 and Ki-67 was performed using the Envision TM (Dako, USA) detection system with antigen recovery. For this, the sections were treated with 3\% hydrogen peroxide $\left(\mathrm{H}_{2} \mathrm{O}_{2}\right)$ diluted in buffered solution for 5 min to block the endogenous peroxide. After recovery of the epitopes, the tissue samples were incubated with primary mouse anti-human ER, PR monoclonal antibody (1:100, 6F11, Novocastra, UK; 1:50; PgR636; DAKO), a rabbit polyclonal anti-Her2 antibody (1:100, cat\# A0485, DAKO) and with mouse anti-Ki-67 monoclonal antibody (1: 600, clone MIB1, DAKO), for a period of $16 \mathrm{~h}$ that included an overnight period in a refrigerator at a temperature of approximately $4{ }^{\circ} \mathrm{C}$. Next, following washing with buffered saline solution, the sections were incubated for 45 min with the Envision TM detection system. To read the reaction, all the sections were treated with a solution of DAB (3,3-diaminobenzidine tetrahydrochloride) at a concentration of $1 \mathrm{mg} / \mathrm{ml}$ of Tris-buffered solution and hydrogen peroxide solution for $5 \mathrm{~min}$. Next, the sections were counterstained with Harris hematoxylin or methyl green for $5 \mathrm{~min}$, followed by dehydration in ethyl alcohol and xylol baths.

The ER and PR immunostained whole-section slides were scored using the Allred scoring system [22]. An Allred score (the proportion score plus the intensity score) of 0 and 2 were interpreted as negative, a score of $\geq 3$ defined ER or PgR positivity $(+)$, a score from $\geq 3$ to $<7$ indicated medium expression $(++)$, and a score of $\geq 7$ indicated rich expression $(+++)$.

The HER2 expression level was classified into four groups according to the scoring guidelines of the HercepTest (Dako) in the set of whole-section slides [23]. HER2 was considered overexpressed with 3+ score positivity and negative with $0+$ or $1+$ immunoreactivity. Tumor blocks with $2+$ score were sent for fluorescence in situ hybridization (FISH) to an independent reference laboratory. For Ki-67 antigen immunostaining, cells were considered positive when the nucleus was stained a brownish color and greater than $1 \%$ of tumor cells. Ki-67 index was calculated as the ratio of Ki-67-positive cancer cells from a total of 1000 cancer cells.

\section{Fluorescence in situ hybridization}

For the analysis of HER2 gene status, FISH assay was performed using FDA approved PathVysion system (Abbott, Downer Grove, IL). Briefly, 4-5 $\mu$ m thick sections were sliced from each tumor block and placed on charged glass slides. After paraffin removal, sections were dehydrated and incubated in $2 \mathrm{mM} \mathrm{NaCN}$ for 20 minutes and later held in pepsin for an average $30 \mathrm{~min}$ followed by denaturation in formamide solution. To each section, $10 \mu 1$ of DNA probe cocktail containing CEP17 (green signal) and LSI HER2 (red signal) was applied, coversliped and sealed for overnight hybridization at $37{ }^{\circ} \mathrm{C}$ in a humidified chamber. Afterwards, slides were washed, counter stained with DAPI and observed for probe signals using a Nikon epi-fluorescent microscope (Nikon, Tokyo, Japan). For scoring, invasive component of the tumor was selected on H\&E stained sections. Red and green signals emanating from the stained sections were counted and interpreted according to the American Society of Clinical Oncology (ASCO) guidelines. A ratio of HER2 to CEP17 of $\geq 2.2$ was considered as HER2 amplified whereas $\leq 1.8$ was interpreted as nonamplified and between 1.8 and 2.2 as equivocal. According to Torrisi and colleagues, polysomy of chromosome 17 was characterized as $\geq 3$ green signals per cell [24].

\section{Treatment plan}

All patients were first diagnosed as advanced breast cancer, and received goserelin (Goserelin Acetate) 3.6 $\mathrm{mg}$ by subcutaneous injection every 4 weeks along with letrozole (Letrozol Tablets) $2.5 \mathrm{mg}$ daily by mouth. If the serum E2 level was not sufficiently suppressed at day 8 after the first dose of goserelin, the administration of letrozole was delayed until the next cycle of goserelin. All patients continued the treatment until progression of disease, the occurrence of a severe adverse event related to the study drug, or withdrawal of consent.

\section{Clinical assessment}

All patients received extensive physical and laboratory examinations, including whole blood cell counts, liver and renal function tests, hormone level tests (E2, FSH, LH), serum CEA, CA125, CA153, brain CT scan, pulmonary CT scan, bone scan, liver CT scan at pretreatment and assessed clinically every 4 weeks for the first 8 weeks of treatment and then every 8 weeks until PD. An additional hormone assay was conducted when patients showed abnormal vaginal bleeding or PD during treatment. Serum E2, FSH, and LH concentrations were measured by chemiluminescent immunoassays using the ADVIA Centaur CP System (Siemens Healthcare Diagnostics, Deerfield, IL). 


\section{Study end points}

The treatment response was evaluated according to the Response Evaluation Criteria in Solid Tumors version 1.0 (RECIST) for measurable lesions and for nonmeasurable variables but evaluable lesions [9].

The primary efficacy endpoint was objective response rate (ORR) and clinical benefit (CB). Objective response rate was defined as the proportion of patients with a confirmed overall response of complete response (CR) or partial response (PR). Clinical benefit was defined as a confirmed assessment of CR, $\mathrm{PR}$, or stable disease (SD) lasting at least 24 weeks. According to the evaluation of measurable lesions, CR was defined as the radiological disappearance of all measurable disease; $P R$ was defined as $\geq 30 \%$ decrease in tumor size or in the sum of all measurable lesions; and SD was defined as a $<30 \%$ decrease or a $<20 \%$ increase in tumor size without the appearance of new lesions. PD was defined as a $\geq 20 \%$ increase in tumor size or the appearance of new lesions. For the evaluation of non-measurable disease such as bone lesions, $\mathrm{CR}$ was defined as the disappearance of all non-measurable lesions; SD was defined as persistence of one or more non-measurable lesions; and PD referred to unequivocal, overall progression of existing non-measurable lesions.

Secondary efficacy endpoints included progressionfree survival (PFS), overall survival (OS) and toxicity. PFS was defined as the time from the start of goserelin with letrozole therapy until objective disease progression or death from any cause. OS was calculated as the period from the first dose of goserelin with letrozole to the date of death or the last follow-up.

\section{Safety assessment}

Safety assessment consisted of monitoring and recording all adverse events and serious adverse events during the treatment and follow-up. The grading of toxicity was recorded according to the National Cancer Institute Common Toxicity Criteria for Adverse Events version 3 (NCI-CTCAE) [10].

\section{Statistical methods}

All statistical analyses were performed using SPSS software version 16.0 (SPSS, Chicago, IL). The study was designed to have a minimal evaluable sample size of 30 patients for the low and unstatistcal incidence of advanced breast cancer in very young women. Baseline patient characteristics were summarized using descrip- tive statistics. PFS and OS were estimated by using the Kaplan-Meier method, and the PFS distributions were compared using the log-rank test. Cox-proportional hazard model was used to assess possible predictive factors for PFS and to estimate the hazard ratios (HR) and $95 \%$ CI. The incidence and severity of toxicities were summarized in standard frequency tables. A two-tailed $P<0.05$ was considered as statistically significant.

\section{Results}

\section{Patients and tumor characteristics}

A total of 35 patients with advanced breast cancer were included in the study (Fig. 1), with median age at diagnosis 32 (range 18-35). 22 patients had not been pregnant, 13 patients had one baby born. 7 patients were obese, while other 28 patients had normal weight. Two patients had family history of breast cancer. The baseline patients characteristics are given in Table 1. All patients had estrogen receptor (ER) and/or progesterone receptor $(\mathrm{PgR})$ positive tumors. Six patients had tumors that were FISH amplified and/or HER2 IHC3+. The median value of Ki-67 index was $29.7 \%$ (range $8-59$ ). All patients achieved amenorrhea after therapy of goserelin and letrozole. The median duration of goserelin and letrozole therapy was 24 (range, 5-37) months, and the median duration of follow-up was 44 (range, 5-79)

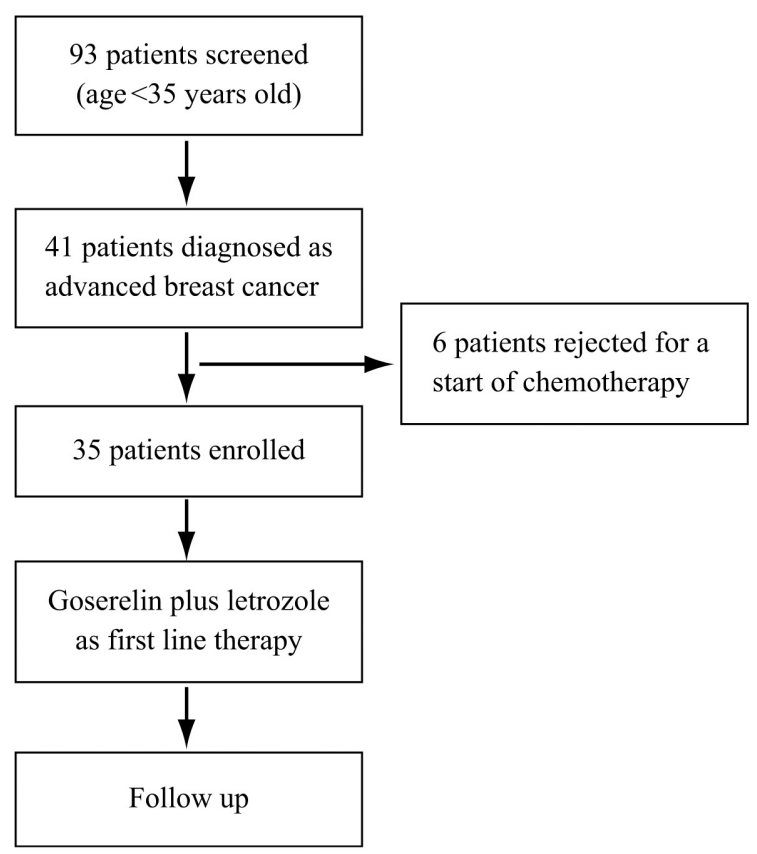

Fig. 1 The treatment procedure for this trial 
Table 1 Characteristics of the patients enrolled in the study

\begin{tabular}{lc}
\hline Characteristics & Number of patients (\%) \\
\hline Median age, years (range) & $32(18-35)$ \\
Number of live birth & \\
0 & $22(62.9 \%)$ \\
1 & $13(37.1 \%)$ \\
$\geq 2$ & 0 \\
Obesity (BMI>28) & \\
Obese & $7(20 \%)$ \\
Normal weight & $28(80 \%)$ \\
Family history of breast cancer & \\
Positive & $2(5.7 \%)$ \\
Negative & $33(94.3 \%)$ \\
ECOG performance status & \\
0 & $4(11.4 \%)$ \\
1 & $21(60 \%)$ \\
2 & $10(28.6 \%)$ \\
Receptor status & \\
ER+PgR+ & $24(68.6 \%)$ \\
ER+PgR- & $6(17.1 \%)$ \\
ER-PgR+ & $5(14.3 \%)$ \\
HER2/neu status & \\
HER2/neu + & $6(17.1 \%)$ \\
HER2/neu- & $29(82.9 \%)$ \\
Ki-67 index & \\
Median &
\end{tabular}

ECOG, Eastern Cooperative Oncology Group; ER, estrogen receptor; PgR, progesterone receptor; HER2/neu, human epidermal growth factor 2; E2, estradiol; SE, standard error; $\mathrm{FSH}$, follicle-stimulating hormone; $\mathrm{LH}$, luteinizing hormone. months. Twenty-three patients continued this therapy until the end of follow-up, other twelve patients discontinued the therapy for progression of disease.

\section{Treatment efficacy}

Goserelin was administered 7 days before the initiation of letrozole treatment. Suppression of serum E2, FSH and LH levels was effectively sustained during gaserelin treatment. All patients were available for evaluation of treatment efficacy.

The median duration of response to the combined endocrine therapy was 21 (range, 10-56) months. The objective response rate was $25.7 \%$ ( $1 \mathrm{CR}$ and $8 \mathrm{PR}$ ). Fourteen additional patients had stable disease at 24 weeks, for a clinical benefit rate of $65.7 \%$. The median PFS was 9.6 (range 5-58) months with 22 (62.8\%) patients free of progression at one year. The other 1/13 patients who occurred disease progression were added with chemotherapy to control disease and the remained 12 patients changed this therapy to chemotherapy. At the time of data cut off, 20 (57\%) patients had died because of disease progression. The median OS was 33 (range 6-72) months (Fig. 2).

We further analyzed the therapy efficacy according to hormone receptor, HER2/neu and body weight status. As showed in Table 2, CB in patients respectively with ER-positive, normal weight and HER2/neu-positive was a little higher than that in patients respectively with ER-negative, obese and HER2/ neu-negtive , but for the limited data, the differences were no statistical.

Univariate analysis showed high Ki-67 index, the presence of liver metastasis and more metastasis sites $(\geq 3)$ were associated with worse PFS ( $\mathrm{HR}=2.93$, $P=0.01 ; \mathrm{HR}=2.07, P=0.03$; HR=1.89, $P=0.04$ respec-

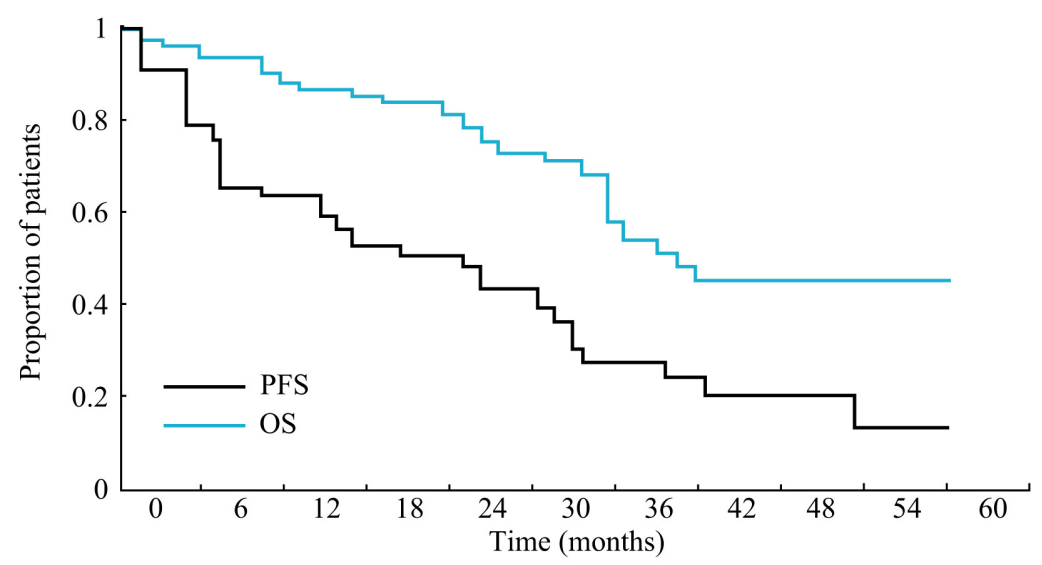

Fig. 2 Progression-free survival (PFS) and overall survival (OS) 
Table 2 Response to therapy of goserelin and letrozole according to Hormone Receptor, HER2/neu and body weight status

\begin{tabular}{|c|c|c|c|c|c|c|}
\hline Status & Total & CR $(\%)$ & PR (\%) & $\mathrm{SD}(\%)$ & $\mathrm{CB}(\%)$ & $\mathrm{PD}(\%)$ \\
\hline \multicolumn{7}{|c|}{ Hormone Receptor status } \\
\hline $\operatorname{ER}(+) \operatorname{PgR}(+)$ & 23 & $1(4.3)$ & $4(17.4)$ & $10(43.5)$ & $14(60.9)$ & $8(34.8)$ \\
\hline $\operatorname{ER}(+) \operatorname{PgR}(-)$ & 7 & - & $2(28.6)$ & $3(42.9)$ & $5(71.4)$ & $2(28.6)$ \\
\hline $\mathrm{ER}(-) \operatorname{PgR}(+)$ & 5 & - & $2(40.0)$ & $1(20.0)$ & $3(60.0)$ & $2(40.0)$ \\
\hline \multicolumn{7}{|l|}{ HER2/neu status } \\
\hline HER2/neu+ & 11 & $1(9.1)$ & $3(27.3)$ & $4(36.4)$ & $7(63.6)$ & $3(27.3)$ \\
\hline HER2/neu- & 24 & - & $5(20.8)$ & $10(41.7)$ & $15(62.5)$ & $9(37.5)$ \\
\hline \multicolumn{7}{|l|}{ Obesity (BMI>28) } \\
\hline Obese & 7 & - & $1(14.3)$ & $2(28.6)$ & $3(42.9)$ & $4(57.1)$ \\
\hline Normal weight & 28 & $1(3.8)$ & $7(25.0)$ & $12(42.9)$ & $20(71.4)$ & $8(28.6)$ \\
\hline
\end{tabular}

$\mathrm{ER}$, estrogen receptor; $\mathrm{PgR}$, progesterone receptor; HER2/neu, human epidermal growth factor 2

Table 3 Predictive factors for progression free survival

\begin{tabular}{lccc}
\hline Factors & HR & $95 \% \mathrm{CI}$ & $P$ \\
\hline Age & 0.73 & $0.47-1.32$ & 0.53 \\
DFI $\geq$ 2 years & 0.95 & $0.53-1.84$ & 0.36 \\
Estrogen receptor - strong positive & 0.81 & $0.35-1.76$ & 0.64 \\
Progesterone receptor - positive & 0.91 & $0.23-2.16$ & 0.76 \\
HER2/neu - positive or negative & 1.03 & $0.51-2.39$ & 0.27 \\
Ki-67 index & 2.93 & $2.35-4.57$ & 0.01 \\
Liver metastasis & 2.07 & $1.34-3.02$ & 0.03 \\
Bone metastasis & 1.32 & $0.64-2.27$ & 0.27 \\
Lung metastasis & 1.37 & $0.89-2.49$ & 0.29 \\
Number of metastasis $\geq 3$ & 1.89 & $1.07-2.84$ & 0.04 \\
\hline
\end{tabular}

HR, hazard ratio; 95\%CI, 95\% confidence interval; DFI, disease-free interval

Table 4 Toxicity of goserelin and letrozole treatment

\begin{tabular}{lccc}
\hline Toxicity & Grade one $(\%)$ & Grade two (\%) & sum (\%) \\
\hline Fatigue & $6(17.1)$ & $1(2.9)$ & $7(20.0)$ \\
Hot flushes & $5(14.3)$ & $2(5.7)$ & $7(20.0)$ \\
Insomnia & $2(5.7)$ & $1(2.9)$ & $3(8.6)$ \\
Headache & $3(8.6)$ & 0 & $3(8.6)$ \\
Weight gain & $3(11.4)$ & 0 & $3(8.6)$ \\
Abdominal pain & $4(11.4)$ & $1(2.9)$ & $5(14.3)$ \\
Nausea & $3(8.6)$ & $1(2.9)$ & $4(11.4)$ \\
Vomiting & $1(2.9)$ & 0 & $1(2.9)$ \\
Lower extremity edema & $3(11.4)$ & 0 & $3(8.6)$ \\
Rash & $3(8.6)$ & 0 & $3(8.6)$ \\
Arthalgias & $2(5.7)$ & $1(2.9)$ & $3(8.6)$ \\
Anorexia & $2(5.7)$ & 0 & $2(5.7)$ \\
Vaginal dryness & $1(2.9)$ & 0 & $1(2.9)$ \\
\hline
\end{tabular}

tively), while hormone receptor, HER2/neu status and other site metastasis did not add benefit to PFS, as showed in Table 3.

\section{Toxicity}

The combined therapy of goserelin and letrozole was well tolerated. All side effects were grade one or two, and were consistent with those known to be associated with goserelin or letrozole monotherapy. No grade three and four toxicities were observed, two patients reported no side effects. Fatigue, hot flashes, abdominal pain and nausea were the more common side effects, showed in Table 4. No patients withdrew from treatment for moderate toxicities. 


\section{Discussion}

Approximately three-quarters of all advanced breast cancers are hormone receptor positive, the mainstay of treatment for these estrogen and/or progesterone receptor-positive breast cancers is endocrine therapy, especially for very young women patients [25]. Temporary and potentially reversible ovarian suppression can be achieved by treatment with a luteinizing hormone-releasing hormone (LHRH) analogue, such as goserelin. Goserelin has shown efficacy for the treatment of premenopausal breast cancer, with equivalent disease-free survival to cyclophosphamide, methotrexate, and fluorouracil chemotherapy in those patients with ER-positive disease [26-28]. Nonsteroidal aromatase inhibitors (AIs), including anastrozole and letrozole, and the irreversible steroidal aromatase inactivator exemestane have demonstrated improved efficacy compared with tamoxifen in the advanced and adjuvant treatment settings $[29,30]$. Therapy of AIs combination with LHRH analogue has been an acceptable option for premenopausal patients with ER and/or PgR positive breast cancer [31].

In our study, we evaluated the efficacy of therapy of goserelin with letrozole for very young women (age $<35$ years) patients with hormone receptor positive advanced breast cancer. Age is an independent risk factor for breast cancer and breast cancer in young women is associated with a poor prognosis. All patients in the present study (median age 32 years) were first diagnosed as advanced breast cancer and chose the therapy of goserelin with letrozole as first-line therapy. The objective response rate was $25.7 \%$, clinical benefit rate was $65.7 \%$ and the median PFS is 9.6 months. Park et al. reported a phase $I I$ study of comparable efficacy between premenopausal metastatic breast cacner patients treated with letrozole plus goserelin and postmenopausal patients treated with letrozole alone as first-line hormone therapy. The ORR, CBR and PFS in premenopausal patients (median age 41 years) was $46 \%, 77 \%$ and 10.9 months, respectively [32]. Yao et al. reported goserelin plus letrozole as first- or secondline hormone treatment in premenopausal patients with advanced breast cancer, their ORR, CBR and PFS in premenopausal patients (median age 40 years) was $21.1 \%, 71.1 \%$ and 10 months [33]. Our result was near but a little lower than these reports, maybe for the younger patients and their aggressive disease. Toxicity of this therapy was not severe, all adverse effects reported were grade one or two. Fatigue, hot flushes and nausea were the reported most common side effects observed among patients treated with goserelin plus letrozole. While there was a little differences among reports, abdominal pain was a more common side effect in our study but not in studies of Park and Yao, arthralgia was also a most common side effect in Park's study but not in studies of Yao and ours. The differences might be due to the colonial and individual difference since the dosage of goserelin and letrozole was same in these reports.

In the present study, $\mathrm{CB}$ in patients with normal weight was higher than that in obese patients $(71.4 \%$ vs $42.9 \%$ ), but for limited data, the difference was no statistical significance. The prognosis in obese patients with breast cancer was poorer $[34,35]$, several models have been suggested to explain the biologic mechanisms. These involve complex relationships between estrogen synthesis, insulin resistance, and altered adipokine and cytokine production [36, 37]. Obese patients have higher levels of estrone, estradiol, and free circulating estradiol and reduced levels of sex hormone binding globulin [38]. In addition, cytokines secreted by the adipocytes can upregulate the aromatase enzyme to further increase the estrogen production, which may stimulate tumor cell growth [39]. Obesity is a component of the metabolic syndrome that includes hyperglycemia, hyperinsulinemia, and insulin resistance. Insulin has mitogenic, anti-apoptotic, and pro-angiogenic properties, and breast cancer cells have been shown to express the insulin receptor [40]. Several studies have demonstrated that hyperinsulinemia is an independent adverse prognostic factor in breast cancer [41-44] and that adiponectin is also related to breast cancer prognosis [45]. Finally, obesity causes subclinical inflammation, increasing the levels of proinflammatory mediators, which may parallel increasing levels of aromatase [46].

In our study, several factors related to PFS identified by Cox model, patients with higher Ki-67 index, presence of liver metastasis and more metastasis sites have poor prognoses. However, no patients were delayed since we changed the endocrine therapy to chemotherapy for patients with disease progression in time.

In summary, goserelin and letrozole can be used as a preference in very young women patients with advanced breast cancer due to the better clinical effect and safety. However, in order to acquire a better therapeutic efficacy, combinations of this therapy with other therapies (such 
as chemotherapy), still need further investigations.

Our study has several limitations. First, due to the very low incidence of breast cancer in very young women, the subjects enrolled in our study were limited to only 35 patients in 4 years, and for some subtypes, the number of patients was really small to draw a firm conclusion. Second, the age of subjects in our study were all below 35 years, all patients had a eager desire to get a therapy which could achieve a better therapeutic efficacy, little adverse effects and more over, a better life quality. So they were willing to enter the study to choose the therapy of goserelin and letrozole as the first-line therapy. For patients who received the therapy and did not get a better clinical efficacy, we promptly changed the therapy to adapted chemotherapy in order to get disease controlled.

\section{Acknowledgements}

We appreciate all subjects for participating in this study.

\section{Disclosure Statement}

No competing financial interests exist.

\section{References}

1. Liukkonen S, Leidenius M, Saarto T, Sjöström-Mattson J (2011) Breast cancer in very young women. Eur $J$ Surg Oncol 37: 1030-1037.

2. Anders CK, Hsu DS, Broadwater G, Acharya CR, Foekens JA, et al. (2008) Young age at diagnosis correlates with worse prognosis and defines a subset of breast cancers with shared patterns of gene expression. J Clin Oncol 26: 3324-3330.

3. Hartley MC, McKinley BP, Rogers EA, Kalbaugh CA, Messich HS, et al. (2006) Differential expression of prognostic factors and effect on survival in young $(<$ or $=40$ ) breast cancer patients: a case-control study. Am Surg 72: 1189-1194; discussion 1194-1195.

4. Holli K, Isola J (1997) Effect of age on the survival of breast cancer patients. Eur J Cancer 33:425-428.

5. Goel S, Sharma R, Hamilton A, Beith J (2009) LHRH agonists for adjuvant therapy of early breast cancer in premenopausal women. Cochrane Database Syst Rev 7: CD004562.

6. Masuda N, Sagara Y, Kinoshita T, Iwata H, Nakamura $\mathrm{S}$, et al. (2012) Neoadjuvant anastrozole versus tamoxifen in patients receiving goserelin for premenopausal breast cancer (STAGE): a double-blind, randomised phase 3 trial. Lancet Oncol 13: 345-352.

7. Gnant M, Mlineritsch B, Stoeger H, Luschin-Ebengreuth G, Heck D, et al. (2011) Adjuvant endocrine therapy plus zoledronic acid in premenopausal women with early-stage breast cancer: 62-month follow-up from the ABCSG-12 randomised trial. Lancet Oncol 12: 631641.

8. Goncalves R, Ma C, Luo J, Suman V, Ellis MJ (2012) Use of neoadjuvant data to design adjuvant endocrine therapy trials for breast cancer. Nat Rev Clin Oncol 9: 223-229.

9. Del Mastro L, Boni L, Michelotti A, Gamucci T, Olmeo $\mathrm{N}$, et al. (2011) Effect of the gonodotrophin-releasing hormone analogue triptorelin on the occurrence of che- motherapy- induced early menopause in pre-menopausal women with breast cancer. JAMA 306: 269-276.

10. Mouridsen H, Giobbie-Hurder A, Goldhirsch A, Thürlimann B, Paridaens R, et al. (2009) Letrozole therapy alone or in sequence with tamoxifen in women with breast cancer. $N$ Engl J Med 361: 766-776.

11. Gerber B, von Minckwitz G, Stehle H, Reimer T, Felberbaum R, et al. (2011) Effect of luteinizing hormone-releasing hormone agonist on ovarian function after modern adjuvant breast cancer chemotherapy. The GBG 37 ZORO study. J Clin Oncol 29: 2334-2341.

12. Sverrisdottir A, Johansson H, Johansson U, Bergh J, Rotstein S, et al. (2011) Interaction between goserelin and tamoxifen in a prospective randomised clinical trial of adjuvant endocrine therapy in premenopausal breast cancer. Breast Cancer Res Treat 128: 755-763.

13. Bartsch R, Bago-Horvath Z, Berghoff A, DeVries C, Pluschnig U, et al. (2012) Ovarian function suppression and fulvestrant as endocrine therapy in premenopausal women with metastatic breast cancer. Eur J Cancer 48: 1932-1938.

14. Ellis MJ, Suman VJ, Hoog J, Lin L, Snider J, et al. (2011) Randomized phase II neoadjuvant comparison between letrozole, anastrozole, and exemestane for postmenopausal women with estrogen receptor-rich stage 2 to 3 breast cancer: clinical and biomarker outcomes and predictive value of the baseline PAM50-based intrinsic subtype-ACOSOG Z1031. J Clin Oncol 29: 23422349.

15. Schwartzberg LS, Franco SX, Florance A, O'Rourke L, Maltzman J, et al. (2010) Lapatinib plus letrozole as first-line therapy for HER-2+ hormone receptor-positive metastatic breast cancer. Oncologist 15: 122-129.

16. Endo Y, Toyama T, Takahashi S, Sugiura H, Yoshimoto N, et al. (2011) High estrogen receptor expression and low Ki-67 expression are associated with improved time to progression during first-line endocrine therapy with 
aromatase inhibitors in breast cancer. Int J Clin Oncol 16: 512-518.

17. Toi M, Saji S, Masuda N, Kuroi K, Sato N, et al. (2011) $\mathrm{Ki}-67$ index changes, pathological response and clinical benefits in primary breast cancer patients treated with 24 weeks of aromatase inhibition. Cancer Sci 102: 858865 .

18. Carlson RW, Theriault R, Schurman CM, Rivera E, Chung CT, et al. (2010) Phase II trial of anastrozole plus goserelin in the treatment of hormone receptor-positive, metastatic carcinoma of the breast in premenopausal women. J Clin Oncol 28: 3917-3921.

19. Pfeiler G, Königsberg R, Fesl C, Mlineritsch B, Stoeger $\mathrm{H}$, et al. (2011) Impact of body mass index on the efficacy of endocrine therapy in premenopausal patients with breast cancer: an analysis of the prospective ABCSG-12 trial. J Clin Oncol 29: 2653-2659.

20. Masuda N, Sagara Y, Kinoshita T, Iwata H, Nakamura $\mathrm{S}$, et al. (2012) Neoadjuvant anastrozole versus tamoxifen in patients receiving goserelin for premenopausal breast cancer (STAGE): a double-blind, randomised phase 3 trial. Lancet Oncol 13: 345-352.

21. Hannouf MB, Brackstone M, Xie B, Zaric GS (2012) Evaluating the efficacy of current clinical practice of adjuvant chemotherapy in postmenopausal women with early-stage, estrogen or progesterone receptor-positive, one-to-three positive axillary lymph node, breast cancer. Curr Oncol 19: e319-328.

22. Harvey JM, Clark GM, Osborne CK, Allred DC (1999) Estrogen receptor status by immunohistochemistry is superior to the ligand-binding assay for predicting response to adjuvant endocrine therapy in breast cancer. J Clin Oncol 17: 1474-1481.

23. Wolff AC, Hammond ME, Schwartz JN, Hagerty KL, Allred DC, et al. (2007) American Society of Clinical Oncology/College of American Pathologists guideline recommendations for human epidermal growth factor receptor 2 testing in breast cancer. J Clin Oncol 25: 118145.

24. Torrisi R, Rotmensz N, Bagnardi V, Viale G, Curto BD, et al. (2007) HER2 status in early breast cancer: relevance of cell staining patterns, gene amplification and polysomy 17. Eur J Cancer 43: 2339-2344.

25. Samphao S, Wheeler AJ, Rafferty E, Michaelson JS, Specht MC, et al. (2009) Diagnosis of breast cancer in women age 40 and younger: delays in diagnosis result from underuse of genetic testing and breast imaging. Am J Surg 198: 538-543.

26. Gerber B, von Minckwitz G, Stehle H, Reimer T, Felberbaum R, et al. (2011) Effect of luteinizing hormone-releasing hormone agonist on ovarian function after modern adjuvant breast cancer chemotherapy: the GBG 37 ZORO study. J Clin Oncol 29: 2334-2341.

27. Karlsson P, Sun Z, Braun D, Price KN, CastiglioneGertsch M, et al. (2011) Long-term results of
International Breast Cancer Study Group Trial VIII: adjuvant chemotherapy plus goserelin compared with either therapy alone for premenopausal patients with node-negative breast cancer. Ann Oncol 22: 2216-2226.

28. Bartsch R, Bago-Horvath Z, Berghoff A, DeVries C, Pluschnig U, et al. (2012) Ovarian function suppression and fulvestrant as endocrine therapy in premenopausal women with metastatic breast cancer. Eur J Cancer 48: 1932-1938.

29. Cuzick J, Sestak I, Baum M, Buzdar A, Howell A, et al. (2010) Effect of anastrozole and tamoxifen as adjuvant treatment for early-stage breast cancer: 10-year analysis of the ATAC trial. Lancet Oncol 11: 1135-1141.

30. Colleoni M, Giobbie-Hurder A, Regan MM, Thürlimann B, Mouridsen H, et al. (2011) Analyses adjusting for selective crossover show improved overall survival with adjuvant letrozole compared with tamoxifen in the BIG 1-98 study. J Clin Oncol 29: 1117-1124.

31. Cheung KL, Agrawal A, Folkerd E, Dowsett M, Robertson JF, et al. (2010) Suppression of ovarian function in combination with an aromatase inhibitor as treatment for advanced breast cancer in pre-menopausal women. Eur J Cancer 46: 2936-2942.

32. Park IH, Ro J, Lee KS, Kim EA, Kwon Y, et al. (2010) Phase II parallel group study showing comparable efficacy between premenopausal metastatic breast cancer patients treated with letrozole plus goserelin and postmenopausal patients treated with letrozole alone as firstline hormone therapy. J Clin Oncol 28: 2705-2711.

33. Yao S, Xu B, Li Q, Zhang P, Yuan P, et al. (2011) Goserelin plus letrozole as first- or second-line hormonal treatment in premenopausal patients with advanced breast cancer. Endocr J 58: 509-516.

34. Protani M, Coory M, Martin JH (2010) Effect of obesity on survival of women with breast cancer: systematic review and meta-analysis. Breast Cancer Res Treat 123: 627-635.

35. Ewertz M, Jensen MB, Gunnarsdóttir Ká, H Højris I, Jakobsen EH, et al. (2011) Effect of obesity on prognosis after early-stage breast cancer. J Clin Oncol 29: 25-31.

36. Grossmann ME, Ray A, Nkhata KJ, Malakhov DA, Rogozina OP, et al. (2010) Obesity and breast cancer: status of leptin and adiponectin in pathological processes. Cancer Metastasis Rev 29: 641-653.

37. Sinicrope FA, Dannenberg AJ (2011) Obesity and breast cancer prognosis: weight of the evidence. J Clin Oncol 29: 4-7.

38. McTiernan A, Rajan KB, Tworoger SS, Irwin M, Bernstein L, et al. (2003) Adiposity and sex hormones in postmenopausal breast cancer survivors. J Clin Oncol 21: 1961-1966.

39. Cleary MP, Grossmann ME (2009) Minireview: Obesity and breast cancer: the estrogen connection. Endocrinology 150: 2537-2542.

40. Vona-Davis L, Howard-McNatt M, Rose DP (2007) 
Adiposity, type 2 diabetes and the metabolic syndrome in breast cancer. Obes Rev 8: 395-408.

41. Goodwin PJ, Ennis M, Pritchard KI, Trudeau ME, Koo J, et al. (2002) Fasting insulin and outcome in earlystage breast cancer: results of a prospective cohort study. J Clin Oncol 20: 42-51.

42. Goodwin PJ, Ennis M, Pritchard KI, Trudeau ME, Koo J, et al. (2012) Insulin- and obesity-related variables in early-stage breast cancer: correlations and time course of prognostic associations. J Clin Oncol 30: 164-171.

43. Pasanisi P, Berrino F, De Petris M, Venturelli E, Mastroianni A, et al. (2006) Metabolic syndrome as a prognostic factor for breast cancer recurrences. Int $J$ Cancer 119: 236-238.

44. Pritchard KI, Shepherd LE, Chapman JA, Norris BD,
Cantin J, et al. (2011) Randomized trial of tamoxifen versus combined tamoxifen and octreotide LAR Therapy in the adjuvant treatment of early-stage breast cancer in postmenopausal women: NCIC CTG MA.14. J Clin Oncol 29: 3869-3876.

45. Duggan C, Irwin ML, Xiao L, Henderson KD, Smith AW, et al. (2011) Associations of insulin resistance and adiponectin with mortality in women with breast cancer. J Clin Oncol 29: 32-39.

46. Morris PG, Hudis CA, Giri D, Morrow M, Falcone DJ, et al. (2011) Inflammation and increased aromatase expression occur in the breast tissue of obese women with breast cancer. Cancer Prev Res (Phila) 4: 10211029. 